\title{
Equatorial plasma bubbles in the ionosphere over Eritrea: occurrence and drift speed
}

\author{
R. H. Wiens ${ }^{1}$, B. M. Ledvina ${ }^{1,{ }^{*}}$, P. M. Kintner ${ }^{1}$, M. Afewerki ${ }^{2}$, and Z. Mulugheta ${ }^{2}$ \\ ${ }^{1}$ School of Electrical and Computer Engineering, Cornell University, Ithaca, New York, USA \\ ${ }^{2}$ Department of Physics, University of Asmara, Asmara, Eritrea \\ *now at: Applied Research Laboratories, University of Texas at Austin, USA
}

Received: 30 November 2005 - Revised: 13 January 2006 - Accepted: 10 January 2006 - Published: 3 July 2006

Part of Special Issue "The 11th International Symposium on Equatorial Aeronomy (ISEA-11), Taipei, May 2005"

\begin{abstract}
An all-sky imager was installed in Asmara, Eritrea $\left(15.4^{\circ} \mathrm{N}, 38.9^{\circ} \mathrm{E}, 7^{\circ} \mathrm{N}\right.$ dip) and used to monitor the OI 630$\mathrm{nm}$ nightglow. Nine months of data were studied between September 2001 and May 2002, a time including the recent maximum in the solar activity cycle. Equatorial plasma bubbles (EPBs) were recorded on $63 \%$ of nights with adequate viewing conditions. The station location within view of the equatorial ionization anomaly and with a magnetic declination near zero makes it an excellent test case for comparison with satellite studies of the seasonal variation of EPBs with longitude. The imager was accompanied by two Cornell GPS scintillation monitors, and the amplitude scintillation data are compared to the all-sky data. GPS scintillations indicate the beginning of EPBs, but die out sooner in the post-midnight period than the larger scale EPBs. Both phenomena exhibit clear occurrence maxima around the equinoxes. Ionospheric zonal drift speeds have been deduced from EPB and GPS scintillation pattern movement. Average near-midnight EPB drift speeds are between 100 and $120 \mathrm{~m} / \mathrm{s}$ most months, with the GPS scintillation speeds being about the same. A winter drift speed maximum is evident in both EPB and GPS scintillation monthly means.
\end{abstract}

Keywords. Ionosphere (Equatorial ionosphere; Ionospheric irregularities) - Radio science (Ionospheric physics)

\section{Introduction}

The study of the equatorial spread F (ESF) phenomenon, great ionospheric turbulence events often detected at latitudes near the magnetic dip equator, has continued for decades and is currently quite intense. The stimuli for current activity are that electron density irregularities associated with ESF interfere with transionospheric radio wave propagation

Correspondence to: P. M. Kintner

(pmk1@cornell.edu) and that its onset remains unpredictable in a strict sense. Two manifestations of ESF make that study accessible to stations that are remote. Plasma instabilities in the lower F region generate bubbles that grow as they rapidly ascend, align themselves with the magnetic field, and generate density irregularities in their wake. The result is a large region of ionization depletion seen from below as one or more darkened flux tubes against the OI 630-nm nightglow background. The nightglow is the result of the two-step process

$\mathrm{O}^{+}+\mathrm{O}_{2} \longrightarrow \mathrm{O}_{2}^{+}+\mathrm{O}$

followed by

$\mathrm{O}_{2}^{+}+e \longrightarrow \mathrm{O}_{2}+h v(630 \mathrm{~nm})$,

so that the absence of ionization, meaning $\mathrm{O}^{+}$ions and electrons, produces an airglow depletion plume usually called an equatorial plasma bubble (EPB). Although too faint to be seen by the naked eye, these bubbles are readily recorded with all-sky imagers fitted with 630-nm interference filters. The second manifestation is the diffraction of transionospheric radio waves by the F-region irregularities. The Global Positioning System (GPS) network of satellites provides all locations on Earth with carefully monitored and coded radio signals from space, which are scattered by the small-scale irregularities associated with ESF. The scattering patterns appear to ground-based receivers as scintillations in the received signal power. Both the EPBs and the GPS scintillations indicate the presence of ESF, and since both are embedded in the background ionization, both move with the drift speed of the ionosphere (Valladares et al., 1996). In the present study we report on observations of both EPBs and GPS scintillations from Asmara, Eritrea $\left(15.4^{\circ} \mathrm{N}, 39.8^{\circ} \mathrm{E}\right.$, $7^{\circ} \mathrm{N}$ dip) made possible by the availability of portable and reliable ground-based instrumentation. This paper concentrates on the EPB observations and uses the accompanying GPS scintillation data primarily for comparison purposes.

Published by Copernicus GmbH on behalf of the European Geosciences Union. 


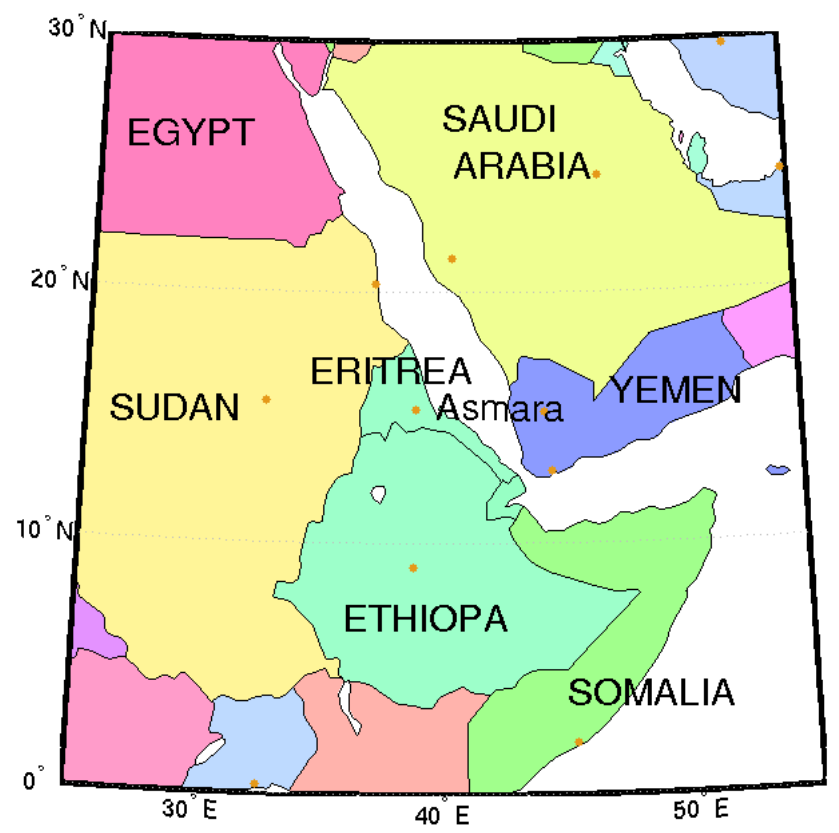

Fig. 1. Regional map of northeastern Africa showing Asmara near the center at $15.4^{\circ} \mathrm{N}, 38.9^{\circ} \mathrm{E}, 7^{\circ} \mathrm{dip}$. The magnetic equator is at $9^{\circ} \mathrm{N}$ in this sector.

Ground-based studies of EPB behavior have been conducted mostly in South America (e.g., Pimenta et al., 2003; Terra et al., 2004), the Pacific Ocean (e.g., Makela et al., 2004), and India (e.g., Mukherjee, 2003), but vast sectors of the tropics have not been and cannot presently be investigated from the ground. Satellite studies (e.g., McClure et al., 1998; Huang et al., 2001) show that the seasonal dependence of EPB occurrence probability is strongly dependent upon longitude, although not all satellites agree on the form of that dependence. Ground truthing at a variety of longitudes should help to resolve some of these differences. This is important because there are several longitude-dependent mechanisms proposed to explain these systematic seasonal variations. The first, by Maruyama and Matuura (1984), supposes that seeding mechanisms are always present at tropical latitudes, but the growth of the Rayleigh-Taylor instability into EPBs is suppressed by transequatorial winds which generally blow from the warm summer hemisphere into the cool winter hemisphere. The second proposal, by Tsunoda (1985), is that the instability grows best when the integrated E-region Pedersen conductivity at the conjugate ends of a flux tube is changing most rapidly, i.e., when the solar terminator is parallel to the magnetic field lines. Burke et al. (2004) point out that the longitude region from $22.5^{\circ} \mathrm{E}$ to $52.5^{\circ} \mathrm{E}$ (where the magnetic declination is essentially zero) at equinox provides both a solar terminator parallel to the field and a minimal transequatorial wind to give maximum EPB occurrence at the equinoxes. Asmara is in this longitude sector and therefore provides a testing ground for these pro- posed influences. McClure et al. (1998) challenged these two hypotheses as primary conditions and suggested that a seeding mechanism dependent upon longitude is available from tropospheric sources. In the event that EPB occurrence probabilities measured at Asmara are not maximum at equinox, a new determinant would be needed, but an equinox maximum would not invalidate the claim made for tropospheric convection seeding. Kil et al. (2004) suggest that the EPB distribution follows the brightness of the OI 135.6-nm emission, proportional to the plasma density, but indicate that none of the mechanisms is successful everywhere at all times.

Ionospheric drift velocities have been measured from EPB motion from the ground (e.g., Terra et al., 2004) and from space (Immel et al., 2003). Most reported measurements are greater than $100 \mathrm{~m} / \mathrm{s}$ in the pre-midnight hours, dropping to around $50 \mathrm{~m} / \mathrm{s}$ as dawn approaches, in agreement with measurements by radio methods, notably those from the Jicamarca incoherent scatter radar (Fejer et al., 1981). These drifts are generally eastward and driven by vertically downward electric fields generated by the nighttime F-region dynamo. Since the occurrence of EPBs is longitude dependent, it is natural to inquire as to the variability of drift speeds with longitude. This is again accomplished by providing observations from as many ground-based stations as possible, and here we offer Asmara data toward this goal.

Since one of the least studied yet potentially most interesting longitude sectors for EPB characterization is northeast Africa, when the opportunity arose for one of the authors to accept a position with the University of Asmara in Eritrea, installing an all-sky imager there became a priority. Asmara is located at $15.4^{\circ} \mathrm{N}, 38.9^{\circ} \mathrm{E}, 7^{\circ} \mathrm{N}$ dip just south of the northern crest of the EIA and its optical counterpart, the northern intertropical arc. The location is at the center of the regional map shown in Fig. 1. The all-sky imager was soon joined by a pair of Cornell University GPS scintillation monitors, which operated there for a total of 22 months. The present paper concentrates on the optical signature of ESF, the EPBs observed with the all-sky imager, and comparisons with the GPS scintillation data for the period in which the scintillation monitors and the imager operated together, from September 2001 until May 2002, a period during which the solar activity cycle reached its maximum. It must be noted that no attempt is made here to separate nights on the basis of any geomagnetic activity index. Our own efforts to do so indicated first that the problem is of sufficient complexity to be deferred to a later paper, and second that, even during this period, the great majority of nights are of low to moderate activity so that the storms only slightly affect the average occurrence statistics.

\section{Instrumentation}

The all-sky imager used in Asmara was borrowed from York University, Canada, where it was constructed and is described by Criswick (1994). Light is collected by a fish-eye 
lens of 8-mm focal length, collimated by a $135-\mathrm{mm}$ telephoto lens, passed through an interference filter optimized for light of 630-nm wavelength, and then focused onto a CCD using a 50-mm imaging lens. All of these lenses are commercial grade for 35-mm format photography. The 50-mm diameter interference filter has a center wavelength of $630.6 \mathrm{~nm}$ and a bandwidth (FWHM) of $1.8 \mathrm{~nm}$ in order to minimize the brightness variation of the $630.0-\mathrm{nm}$ nightglow radiation over the $0^{\circ}$ to $5^{\circ}$ incidence angles set by the lens geometry, i.e., to optimize the "flat-field" characteristic. This filter is carried on a wheel, along with seven others, which is driven by a stepping motor. The detector is a Kodak KAF1300 CCD with $1300 \times 102816 \times 16 \mu \mathrm{m}$ pixels, cooled to $-30^{\circ} \mathrm{C}$. Elementary pixels are binned at the camera into $4 \times 4$ subarrays so that images are transferred to the accompanying personal computer as $256 \times 256$ arrays and stored in 16-bit FITS format for easy viewing with Smithsonian Astrophysical Observatory software.

The all-sky imager was operated on the roof of the main University of Asmara building near the center of the city but well above it so that city lights were not a severe problem for the present purposes. On and off times were programmed to limit observations to solar elevation angles less than $-12^{\circ}$ and lunar elevation angles less than $+5^{\circ}$ or lunar disk illumination less than $60 \%$. Three positions of the filter wheel constituted the normal operational cycle: one for measuring the OI 630$\mathrm{nm}$ red line nightglow and two for the two wavelengths for monitoring background light, assumed to be white. Each filter was in place for a 60-s exposure so that the time between successive red line images was $216 \mathrm{~s}$. The main rainy season in Asmara is during the northern hemisphere summer, so data from June through August are unavailable. At other times clouds occur, but many sequences of cloud-free nights made it an above-average site. Results of an analysis of red line brightness distribution in latitude and time using this data set are described by Wiens et al. (2004).

The Cornell GPS scintillation monitors also operated on the roof of the main University of Asmara building separated along a magnetically east-west line by $63 \mathrm{~m}$. They are based on a GEC Plessey development system modified for logging the L1-band $(1.57542 \mathrm{GHz})$ GPS signal power at a rate of 50 samples/s. Each unit is contained on one printed circuit board mounted in a personal computer and is attached to a $10-\mathrm{cm}$ diameter powered patch antenna. Design, construction, and testing details are given by Beach and Kintner (2001).

\section{Observations}

\subsection{EPB occurrence}

An all-sky image in 630-nm light is shown in Fig. 2. North is upward and east is to the right. The outer edge of the disk is the horizon and zenith is in the center of the disk. A pair

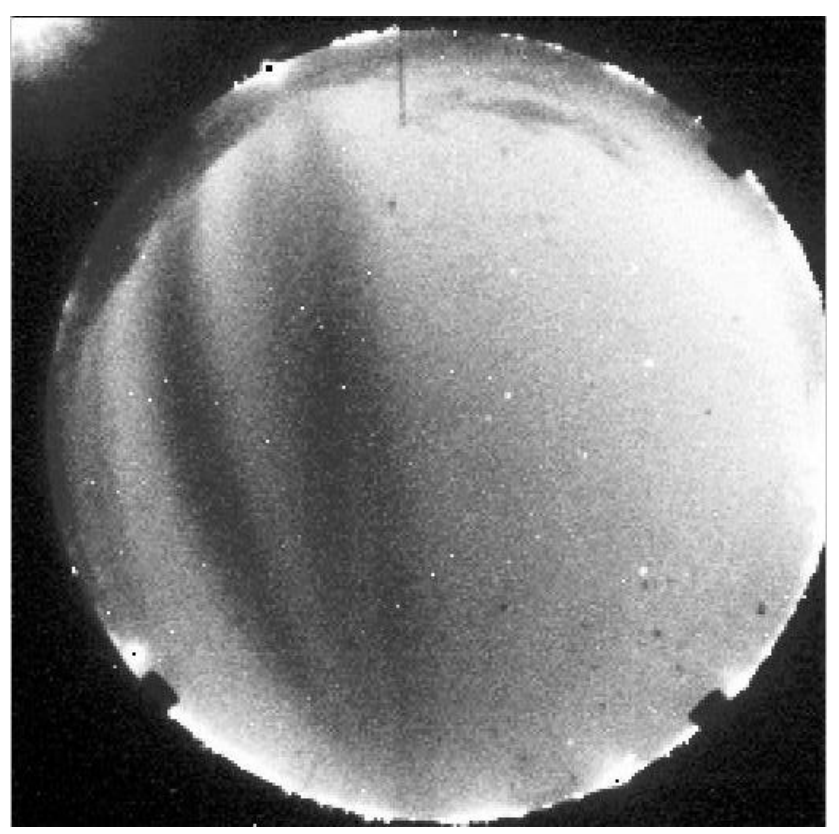

Fig. 2. Typical all-sky image over Asmara in late morning. The outer edge of the disk corresponds to the horizon and the center to zenith. North is to the top and east is to the right. The large black streaks are EPBs.

of EPBs is seen crossing the disk from left to right aligned along the geomagnetic field direction. EPBs are often as well-defined and readily detectable as these, but at least as often they are faint or absent. Our first task was therefore to seek a climatology of occurrence. Each image was assigned a value of 1 for EPB visually detected anywhere on the disk, 0 for no EPB seen in spite of acceptable observing conditions, and 2 for unacceptable conditions, clouds, excessive moon, or instrumental problems. These values were grouped into time intervals of $0.2 \mathrm{~h}(12 \mathrm{~min})$ duration. If a time sample included one image that showed an EPB, the whole interval was deemed a 1 . The matrix of these values is plotted on a day number vs. local time grid in Fig. 3.

The first characteristic one notices in Fig. 3 is that vertically there are nine major groups of data separated by grey areas, indicating no data available. The groups correspond to months, beginning with September 2001 and ending with May 2002. The data gaps are periods when the moon was a hindrance. More importantly, Fig. 3 shows a black interval from day 106 (15 December) to day 156 (3 February), indicating an almost total absence of EPBs centered on 9 January (day 130), twenty days after the winter solstice (day 110). Data groups above and below this show the appearance of white bands, indicating EPB much of the night. The autumnal equinox, SE, on day 21 is white and is surrounded by nights of EPB sighting. The vernal equinox, ME, on day 202 coincides with the moon interfering with evening observations, but the data groups before and after that time also 


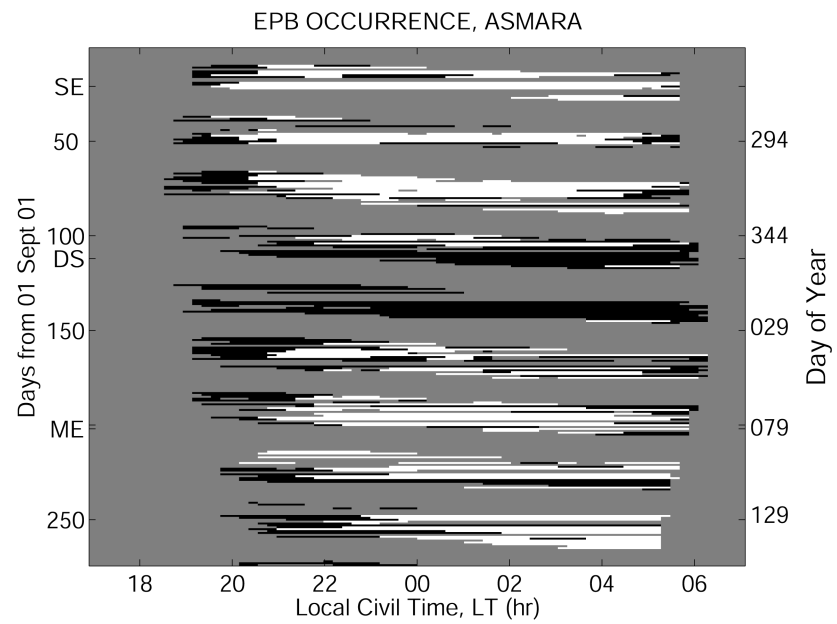

Fig. 3. Daily occurrence record of EPB over Asmara. White indicates EPB seen in at least one image during a 12-min interval; black is no EPB sighted in spite of favorable viewing conditions; and grey indicates unfavorable viewing, moon, or clouds. $\mathrm{SE}=$ September equinox, $\mathrm{ME}=$ March equinox, and $\mathrm{DS}=$ December solstice.

indicate much EPB activity. Figure 3 demonstrates that at Asmara EPBs are most frequent around the equinoxes. There is also considerable activity after the equinoxes, and a suggestion that the activity center may lie as much as twenty days after the actual equinoxes at days 41 (11 October) and 222 (10 April). The monthly statistics of EPB occurrence are listed in Table 1. If any image during the night showed an EPB, it was counted as an occurrence. If all cloudless periods during a night, no matter how brief, showed no EPB, then the night was characterized as zero occurrence. The table shows remarkably high occurrence rates for this period, which, as already indicated, is at the maximum of the solar activity cycle.

Another striking feature of Fig. 3 is that EPBs are first sighted during the evening hours and often continue into the late morning hours. Caution must be exercised in considering the notable exceptions after 9 April (day 221) and especially the bottom group representing all of May. This visual method of detecting EPBs depends upon contrast between the EPB and its airglow background. As shown by Wiens et al. (2004), the evening EIA disappears over Asmara in April and May. This seasonal change occurs because ionization produced by the fountain effect is pushed by a transequatorial neutral wind that blows from the warm summer hemisphere into the cool winter hemisphere. This neutral wind pushes ionization down the magnetic field lines for faster recombination and increased nightglow brightness in winter, and up the field lines for slower recombination and reduced optical emission in summer. The transition occurs at Asmara in April and affects the premidnight brightness because the fountain effect is strong only in the evening. After midnight the nightglow brightness is governed by the midnight pres-
Table 1. Monthly occurrence frequency.

\begin{tabular}{lrrrrrr}
\hline & \multicolumn{3}{c}{ Total Nights } & \multicolumn{2}{c}{ Event Nights } & \multicolumn{2}{c}{ Occurrence } \\
Month & EPB & GPS & EPB & GPS & EPB & GPS \\
\hline 1 Sep & 13 & 28 & 13 & 28 & 100 & 100 \\
1 Oct & 11 & 31 & 8 & 25 & 73 & 81 \\
1 Nov & 21 & 30 & 19 & 25 & 90 & 83 \\
1 Dec & 21 & 29 & 6 & 7 & 29 & 24 \\
2 Jan & 17 & 31 & 1 & 10 & 6 & 32 \\
2 Feb & 19 & 28 & 13 & 21 & 68 & 75 \\
2 Mar & 22 & 29 & 15 & 26 & 68 & 90 \\
2 Apr & 16 & 29 & 11 & 29 & 69 & 100 \\
2 May & 19 & 31 & 14 & 28 & 74 & 90 \\
\hline
\end{tabular}

sure bulge, a tidal effect shown by Wiens et al. (2004) to be effective during April and May as well as during the winter months. This gives the April and May data a bias toward post-midnight detection, to the extent of EPBs being recognizable until dawn. Therefore we know that EPBs are common over Asmara in May, but we are unable to reliably estimate their starting time. For the rest of the year, when EPBs occur they usually begin between 20:00 LT and 22:00 LT and continue into the late morning hours.

It is instructive to compare the occurrence of EPBs with the occurrence of GPS scintillations recorded by the scintillation monitors in Asmara over the same period of time. The $\mathrm{S}_{4}$ index, the unity-normalized standard deviation of signal power (Yeh and Liu, 1982), is calculated from the 50-Hz signal power time history for every minute of monitor operation for every GPS satellite above the $30^{\circ}$ elevation angle. In Asmara the number of GPS satellites available at any time varied between six and ten, giving excellent coverage over the whole sky and ideal for comparison with the all-sky images. The maximum $\mathrm{S}_{4}$ from all satellites in a 12-min interval was selected to represent that interval. The night-by-night, hourby-hour results are plotted on a local time vs. day number grid in a manner similar to that used for the imager data in Fig. 3. The plot shows the same 274 days in which the GPS measurements were accompanied by all-sky images, and all intervals with a maximum $\mathrm{S}_{4}$ index greater than or equal to 0.4 are assigned the value 1 ; all smaller $S_{4}$ indices are given the value 0 . The plot is given in Fig. 4, specifically for comparison with Fig. 3. The obvious benefit of using radio methods rather than optical is the greatly reduced operational gaps because the GPS measurements are unaffected by cloud and moonlight. The data gaps that do appear are usually the result of power failures, from which the monitor automatically recovers, or operator absences, which were infrequent. A number of diagonal streaks pointing upward toward the right are the effect of multipath interference, their direction showing the change of sidereal time with respect to solar time, and are not related to ionospheric effects. 


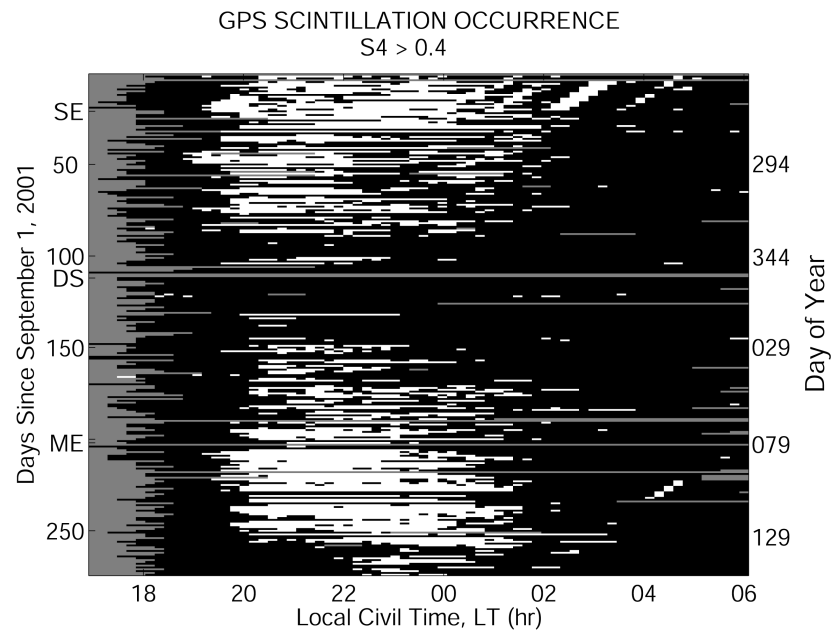

Fig. 4. Daily occurrence record of GPS scintillation with $S_{4}>0.4$. White indicates occurrence for any fraction of a 12-min time interval, black is no such occurrence, and grey indicates no operation.

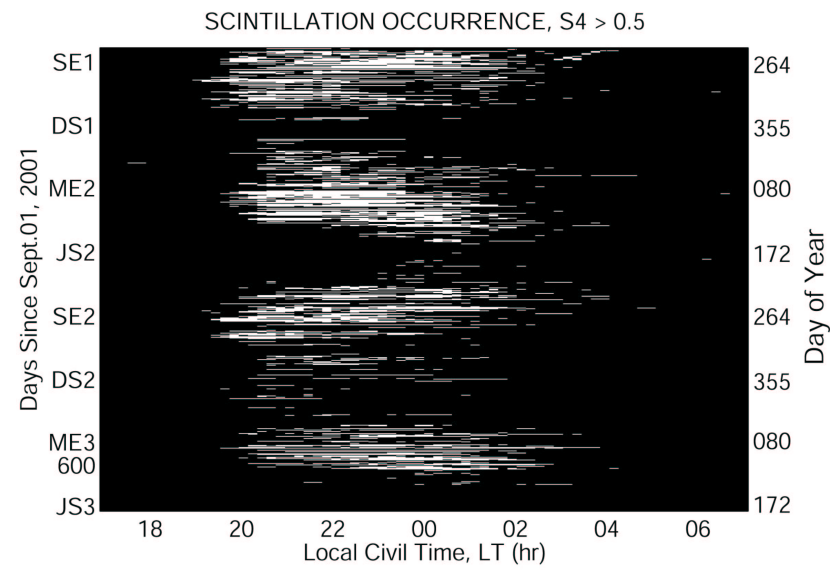

Fig. 5. Daily occurrence record of GPS scintillation with $S_{4}>0.5$ for a full 22 months to illustrate equinox symmetry. The integer following SE, DS, etc., gives the year, e.g., "SE2" = "September equinox 2002".

The GPS scintillation occurrence pattern in Fig. 4 shows the same seasonal variation as we see in Fig. 3, featuring the same dark band around day 110, the winter solstice, and maximum activity levels near the equinoxes, SE and ME. Figure 4 would agree with Fig. 3 in centering the equinoctial activity around days 41 and 222, twenty days after the actual equinox dates, but the solstice gap in this case does not show that 20-day lag. The solstice gap appears to show a greater likelihood of GPS scintillation (at the $\mathrm{S}_{4}>0.4$ level) than of EPB appearance. The monthly summaries of occurrence frequency are listed in Table 1 , again choosing $\mathrm{S}_{4}$ at the 0.4 threshold.

Having demonstrated that the EPB and the GPS scintillations both show maxima at the equinoxes, it would be worth-
Table 2. Monthly occurrence frequency.

\begin{tabular}{lrr}
\hline Month & \multicolumn{2}{c}{$\%$ Occurrence } \\
& AGPS & DMSP* \\
\hline January & 11.3 & 1.2 \\
February & 14.5 & 11.0 \\
March & 39.0 & 25.9 \\
April & 50.8 & 44.3 \\
May & 9.8 & 13.4 \\
June & 0.0 & 5.2 \\
July & 0.0 & 3.3 \\
August & 19.2 & 23.4 \\
September & 51.8 & 36.7 \\
October & 35.6 & 25.3 \\
November & 30.0 & 14.2 \\
December & 6.9 & 0.0 \\
\hline
\end{tabular}

* from L. C. Gentile (personal communication)

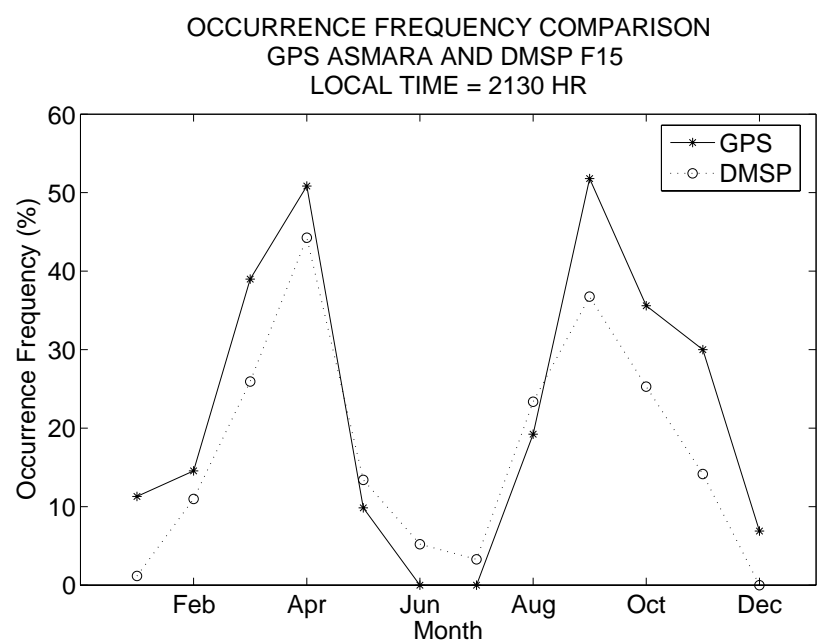

Fig. 6. Monthly average occurrence frequency comparison of Asmara GPS scintillation and DMSP EPB detection. Points are given at the start of the month for the whole month. The figure shows both satellite and ground-based agreement on the symmetry of equinoctial maxima.

while to determine whether the solstice occurrences are also symmetrical. For this we invoke the longer and more complete record of GPS scintillation occurrence shown in Fig. 5, also as an occurrence image on a local time vs. day number grid.

In Fig. 5 there are four distinct equinox groupings corresponding to the September 2001 (SE1), the March and September 2002 (ME2 and SE2), and the March 2003 (ME3) equinoxes, separated by the adjacent solstice periods. The 22-month record shown here allows for 12 monthly averages of occurrence probability to be calculated as shown in Table 2, chosen specifically for a local time of 21:24 LT to 21:36 LT. These data are also plotted in Fig. 6, which shows 
Table 3. Width increase of EBPs.

\begin{tabular}{lrrrr}
\hline & 17 Nov & 17 Feb & 19 Feb & 12 May \\
\hline Local Time (h) & 26.57 & 24.53 & 26.66 & 26.21 \\
EPB Width (km) & 110.06 & 53.37 & 223.46 & 163.43 \\
Growth Rate (m/s) & 10.0 & 14.0 & 7.7 & 7.6 \\
Exp Doubling (h) & 2.44 & 1.00 & 5.73 & 5.09 \\
\hline
\end{tabular}

clear and almost equal maxima for April and September. The minimum in June and July, with zero occurrences, is slightly deeper than the December/January minimum with occurrence frequency only as low as $6.9 \%$. Figure 6 shows the two halves of the yearly cycle as being similar.

The primary hours of GPS scintillation activity are indicated by the horizontal extent of the white bars in Fig. 4 . Most of the scintillation occurs between the hours of 20:00 LT and 02:00 LT. This is quite unlike the EPB behavior, which usually continues into the late morning hours and has implications we discuss later. As discussed by Makela et al. (2004), the morning extension of the GPS scintillation traces increases with a decreasing $\mathrm{S}_{4}$ index, so $\mathrm{S}_{4}$ indices around 1.0 rarely occur after 00:00 LT and those of 0.15 and above continue until morning, just like the EPB occurrences. The threshold of $\mathrm{S}_{4}=0.4$ was selected for presentation here because of a perceived coincidence between the earliest ever first sighting of EPB activity in the evening and the first appearance of $\mathrm{S}_{4}$ rising above that level that same evening. Comparing the start times of the two phenomena in Figs. 3 and 4 shows that usually the GPS scintillation reaches $S_{4}=0.4$ before EPBs are visually detected. As described in the following section, EPBs are difficult to detect in the formative stage. Both figures show that the earliest start times occur at the equinoctial maxima of activity. Start times are around 20:30 LT nearest the solstice and about an hour earlier at the height of the activity season, a trend admittedly easier to see in Fig. 4 than in Fig. 3. Notice that the late start of EPBs in May receives some confirmation from the late start of GPS scintillations in late May as well.

\subsection{EPB morphology}

Given the unique geographical location and the frequent occurrence of EPB over Asmara, it is natural to ask whether this data set can describe the origin, the fate, and the nightly behavior of the EPBs. Their usual fate is the easier challenge because they are often seen to decay and disappear when viewing conditions are favorable. In the morning hours EPBs are usually seen to move into the field of view from the western horizon, often individually, but just as often in groups of two or more in succession, as in Fig. 2. They apparently expand as they approach the zenith of the all-sky image. Part of this apparent expansion is simply that equal arc lengths in the sky project to shorter horizontal ground distances near the zenith than at smaller elevation angles. However, real growth in width is also evident. The growth of four well-defined EPBs has been estimated by determining their widths (projected onto the ground) near the horizon and again as late as we could still estimate edges. The widths and growth rates are summarized in Table 3 . The narrowest we measured was $53 \mathrm{~km}$ wide but all were less than $250 \mathrm{~km}$. If it is assumed that the width expands exponentially and we use the relationship:

$\tau=\frac{\log (2)}{\log \left(w_{2} / w_{1}\right)} \Delta t$,

where $w_{1}$ and $w_{2}$ are respectively the initial and final width measurements, and $\Delta t$ is the time between these measurements, then $\tau$ is the exponential doubling time given in Table 3. This ad hoc exponential doubling time is from 1 to 6 hours according to these four EPBs. Alternatively, the linear growth rate, listed simply as the growth rate in Table 3, may be a more realistic parameter: that mean is $9.8 \mathrm{~m} / \mathrm{s}$. As the EPBs expand, the forces that shut out ionization weaken and an inward diffusion of ionization ensues, causing them to fade. Some survive to cross the eastern rim of the image, but others fade out of existence beforehand.

The period from 19:30 LT to about 23:00 LT provides strikingly different behavior. In Asmara the EIA is prominent but north of the station, a crescent-shaped brightening along the top of the disk is not seen in Fig. 2. The first appearance of EPBs is as small, closely spaced streamers more or less confined to the EIA crest region and perpendicular to it. These streamers are invariably moving eastward, often from the western extreme of the crescent, but sometimes arising from areas of the crescent well to the right of the western extremity, possibly east of the crescent center. These latter streamers we suppose to be formed within our field-of-view. These cases cannot be illustrated here because they are detectable only by playing a movie of successive images. EPBs in this time period are seen in varying widths, indicating that some are first seen at a later stage of development than others. We therefore claim that northeastern Africa can be either a source region or slightly east of a source region for EPBs. After this period and into the morning there is no evidence for incipient EPBs; all come from the western horizon.

\subsection{EPB drift speed}

The previous section indicated that the EPBs are always in motion. It is possible to estimate the speed of the drift by comparing the location of an EPB on successive images and simply dividing by the time difference between them. To simplify the data processing, a west-east linear array of pixels including zenith has been used to estimate the drift speed. Pixel position in the array is linearly related to zenith angle and can be mapped to a ground location. This mapping assumes a red line emission altitude of $280 \mathrm{~km}$. The projected 
pixel array is $882 \mathrm{~km}$ centered on the station. Brightnesses recorded in the pixel coordinate system are interpolated to a scale of 2-km intervals on the distance coordinate. In principle the linear arrays of brightness for successive images can be cross-correlated, the correlation delay giving the distance the features have moved. Greater success was achieved, however, by applying increasing delays until the horizontal distances between features were minimized, the amount of shift required providing the distance moved. This method worked best when the EPB had well-defined edges and when adjacent frames had been used. But, as shown in the all-sky images of Makela et al. (2004), EPBs are constantly in turbulent motion and, as found here, they both grow as they move and the perspective of their three-dimensional geometry changes. Thus, successive images frequently differ enough that the analysis program could be confounded. Instead, the procedure employed uses nine images in eight successive pairs, rejecting by visual inspection any of the fits that are regarded as unsuitable, and averaging the rest over the half hour the nine images represent. Monthly hour-by-hour averages have been compiled and plotted as a series of nine monthly graphs in Fig. 7. Error bars are standard deviations, including the variability of the measurement technique and the natural variability.

The most common feature of the average nightly variation of EPB drift speed shown in Fig. 7 is a decrease throughout the morning hours. Some seasonal variation is evident in that the months of October through February show drift speeds greater than $100 \mathrm{~m} / \mathrm{s}$ most of the night. March and September drifts reach speeds faster than $100 \mathrm{~m} / \mathrm{s}$ only in the pre-midnight hours, and April and May drifts are almost always less than $100 \mathrm{~m} / \mathrm{s}$. The hourly averages for the 03:00 LT hour are plotted in Fig. 8, which shows a winter maximum, even though the data from December and January, months 4 and 5 , are quite limited.

For comparison purposes, the drift speeds deduced from the GPS scintillations are shown for the same set of months in Fig. 9. The speed is measured by correlating the $50-\mathrm{Hz}$ received signal power time histories from the two GPS receivers that they receive from one GPS satellite. With a 63-m separation along a magnetic east-west line, the zonal drift speed is calculated as this distance divided by the crosscorrelation delay time. The method is described in Ledvina et al. (2004). Figure 9 shows that the zonal drift speeds deduced from the GPS scintillations seem to decrease more rapidly through the morning hours than those found from the EPB measurements (Fig. 7) but, in general, the scintillation determinations are of the same magnitude as the EPB determinations. The winter maximum of zonal drift speeds suggested by the EPB measurements is verified by the GPS scintillation measurements. In Fig. 8 the average monthly drift speed from Fig. 9 at 00:00 LT is plotted against month, showing a pronounced winter maximum similar to the EPB plot for 03:00 LT in the same figure.

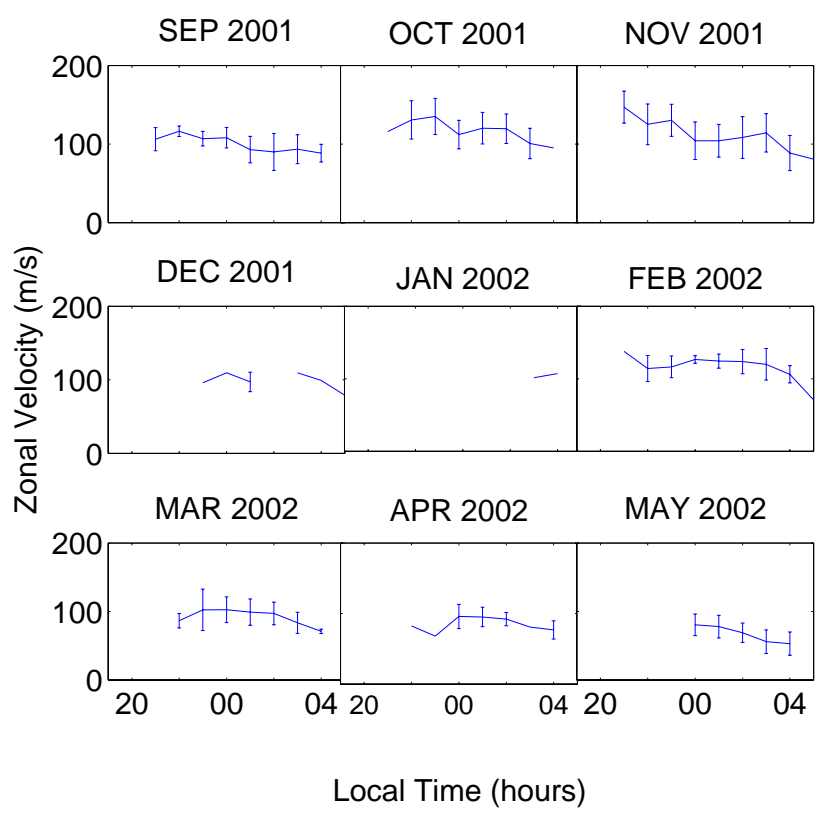

Fig. 7. Monthly means of hourly EPB drift velocity. Error bars indicate standard deviations including measurement and geophysical variability; lack of an error bar indicates fewer than three data.

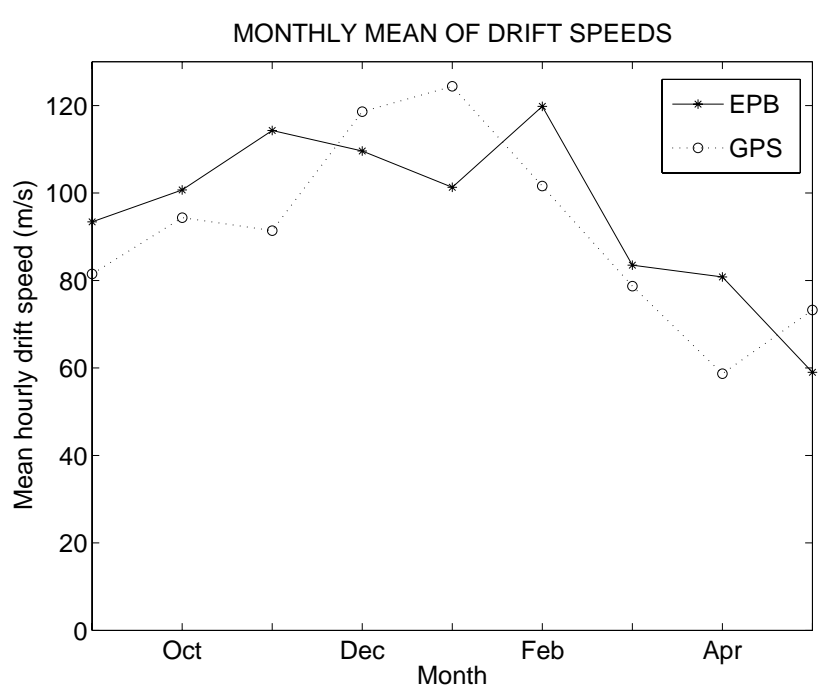

Fig. 8. Annual variation of zonal ion drift speed from EPBs (solid) and from GPS scintillation (dashed) measurements. The EPB trace uses 03:00 LT averages from Fig. 5 because they are available for all months. The GPS scintillation trace is for midnight averages. Both show the winter maximum. 


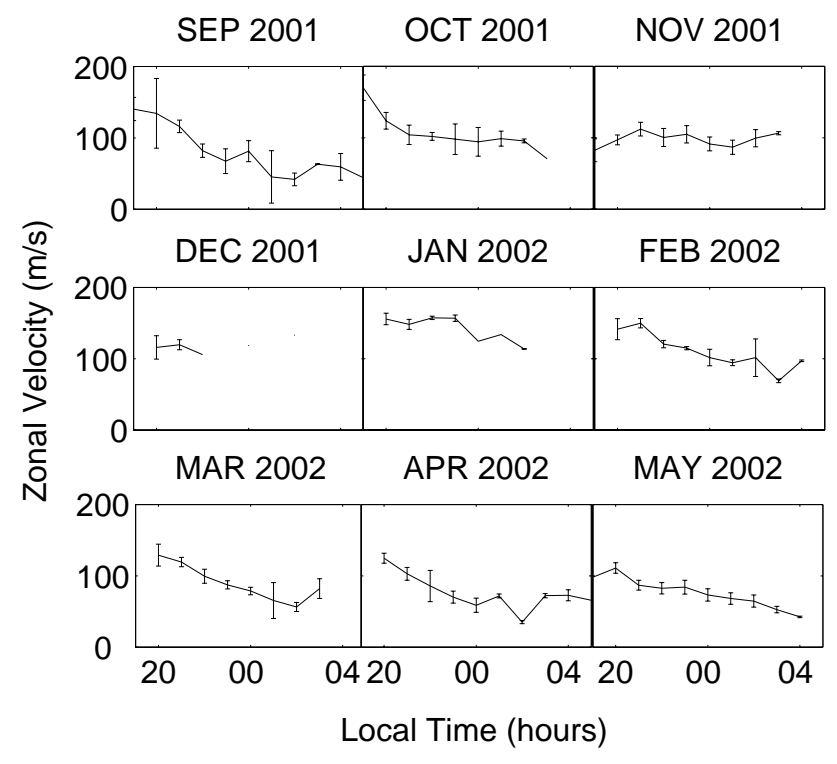

Fig. 9. Monthly mean of hourly GPS scintillation drift velocity. Error bars indicate standard deviations, including measurement and geophysical variability.

\section{Discussion}

\subsection{Occurrence probability}

\subsubsection{Annual variation}

A recent ground-based study of EPBs readily comparable to and overlapping this data set in time was made by Makela et al. (2004) from Haleakala, Hawaii $\left(20.7^{\circ} \mathrm{N}, 203.7^{\circ} \mathrm{E}\right.$, geomagnetic $21.3^{\circ} \mathrm{N}$ ). They employed an all-sky imager sensitive to OI 630.0-nm nightglow and a narrow-field camera sensitive to OI 777.4-nm nightglow. One main difference between their station location and Asmara is that they are north of the EIA crest. The easiest comparison is between Table 1 and Makela et al.'s Table 2. First of all, notice that Table 1's occurrence probabilities are somewhat larger than theirs, varying from $6 \%$ in January to $100 \%$ in September, while theirs are between $0 \%$ in December and January to $41 \%$ in September. This may result from the Hawaiian camera looking at the flux tubes from the top side (tangent to the magnetic field) north of the EIA, while the Asmara camera, south of the EIA crest, views them from below, making it less sensitive to azimuth variation. Also notice that the Hawaiian September maximum is much broader than the April maximum. The Asmara EPB data alone cannot dispute such broadening because of the lack of July and August imager data, but Asmara's March maximum is broader than that in Haleakala, showing abundant EPBs from February to May. More significantly, Fig. 6, using Asmara GPS scintillation data, shows both equinox maxima to be about the same width. Makela et al. (2004) caution that their image data around the April maximum may have cloud problems, but they are supported by the GPS scintillation occurrence probabilities. The differences in the seasonal variation of EPB occurrence between Asmara and Hawaii are real and substantial due to the differences between the magnetic latitudes and the longitudes of the two stations.

The symmetry of the equinox occurrence maxima is supported by the DMSP F15 measurements of ionospheric depletion reported by Gentile et al. (2006). The DMSP occurrence probabilities reported for the longitude range of $30^{\circ}$ to $45^{\circ}$ at the local time of 21.3 to 21.5 MLT for the period of 2000 to 2004 binned according to month are listed in Table 3 and plotted in Fig. 6 along with our Asmara GPS scintillation monthly occurrence probabilities. The satellite data clearly show two equinox maxima coincident with the Asmara data at April and September with deep minima at the solstices. A small difference is that the DMSP December-January minimum is deeper than the June-July minimum, the opposite of the comparison for the Asmara GPS scintillations, but this may result from the small number of occurrences involved. Since the DMSP satellites measure EPBs above $800 \mathrm{~km}$ and the Asmara imager measured them below $300 \mathrm{~km}$, the comparison is not exact. Nevertheless, it may be supposed that a certain fraction of EPBs found at $300 \mathrm{~km}$ will actually reach $800 \mathrm{~km}$, as indicated by Gentile et al. (2006).

As stated earlier, the Asmara seasonal occurrence probability may provide a test for the success of proposed mechanisms used to explain seasonal/longitudinal dependence globally. Burke et al. (2004) pointed out that, at the Asmara longitude (between $22.5^{\circ}$ and $52.5^{\circ}$ ), the conditions set by both Maruyama and Matuura (1984) and by Tsunoda (1985), i.e., minimal suppressive transequatorial wind and solar terminator parallel to the magnetic field, hold true at the equinoxes, producing maximum EPB occurrence then. Our data in Figs. 3 through 6 and in Tables 1 and 2 show definitively that this is the case. McClure et al. (1998) also studied the longitude dependence of EPB occurrence, this time using data from AE-E in situ satellite measurements over four seasons. They challenged the Maruyama and Matuura (1984) proposal on the basis of longitude comparisons of EPB occurrence with several other studies. Here the comparisons with Maruyama and Matuura (1980), Watanabe and Oya (1986), and McClure et al. (1998) lend themselves to a comparison study with our ground-based data. Ample justification for the validity of comparing these diverse satellite measurements is given by McClure et al. (1998), and we again suppose some direct relationship between the satellite and ground-based measurements of occurrences. Occurrence probability vs. longitude plots are given by McClure et al. (1998) for each of the satellite studies for four seasons: February, March, April; May, June, July; August, September, October; and November, December, January. Each season's graph has been scaled for each of the satellite studies presented by McClure et al. (1998) at a longitude of $+40^{\circ}$, and then plotted along with our own data in Fig. 10. In the case 
of Asmara's data, labelled "Asmara" in Fig. 10, the values from Table 1 have been grouped together into these threemonth groups. Asmara's occurrence probabilities are much higher than those from the three satellite studies. For easier comparison, Asmara's data are plotted using the ordinate axis on the right side of the plot. In Fig. 10 the seasonal variation at Asmara appears to be of the same form as that of McClure et al. (1998) and least like that of Maruyama and Matuura (1980). However, our EPB representation for the season May-June-July is restricted to data for May. GPS scintillation data from May, June, and July (Table 1) give only a $3.3 \%$ probability of occurrence, which lowers the second Asmara point so that the form resembles the Maruyama and Matuura (1980) trace. The Asmara data thus do not contradict any of the hypotheses given, although they readily lend confirmation to the equinox EPB maxima predicted by the Maruyama and Matuura (1984) and the Tsunoda (1985) mechanisms. Some of the difference between the seasonal variation patterns at Asmara and Haleakala could be due to the fact that Haleakala is in a maritime environment while Asmara's environment is continental, the tropospheric seeding mechanism of McClure et al. (1998) thus affecting the two environments differently. As pointed out by Kil et al. (2004), the global longitude/seasonal distribution of EPBs will not be completely explained by only one mechanism but by a number of mechanisms. They find a correlation between the brightness of the OI $135.6 \mathrm{~nm}$ radiation (related to F-region plasma density) seen by the TIMED/GUVI satellite imager and point out that, like all other correlations, this one has its limits of applicability.

\subsubsection{Diurnal variation}

A comparison of Figs. 3 and 4 shows that the GPS scintillations reach $\mathrm{S}_{4}>0.4$ at least as early as the first visual detection of EPBs. Usually a half hour or so is needed for the disturbance to grow from the 250 -m scale size that causes the GPS scintillation to the tens of kilometers characteristic of detectable EPBs. For the rest of the evening, the two scale sizes of the ESF coexist. The fact that the GPS scintillations cease after 02:00 LT while the EPBs persist results from the small-scale irregularities disappearing earlier than the large-scale irregularities and from the general decrease in ionization in the morning hours. This same behavior was reported by Makela et al. (2004) in Hawaii. They were able to watch the initial growth of the ESF plumes near sunrise at the equinox maxima. The first appearance of EPBs after 22:00 LT was common for dates not near the equinox maxima, as is true in our data. These EPBs showed no sign of having developed near the station and are attributed to origins west of Hawaii. The same effect is seen in Asmara by noting that late beginnings always came from the western horizon while early beginnings could sometimes be located at the longitude of Asmara and even to the east. Makela et al. (2004) report some EPBs arriving over Haleakala as

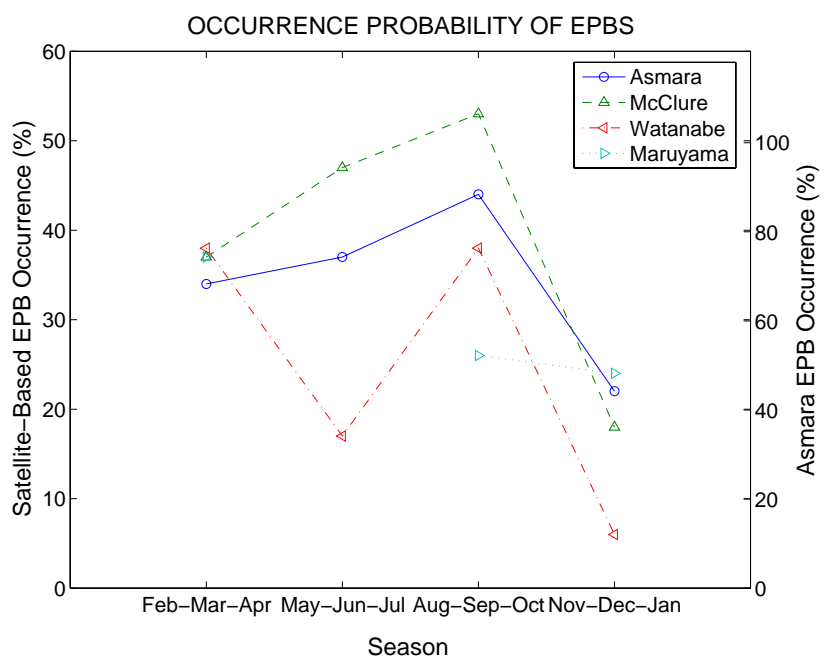

Fig. 10. Seasonal variation of occurrence probability of EPBs at $40^{\circ}$ comparing Asmara with McClure et al. (1998), Watanabe and Oya (1986), and Maruyama and Matuura (1980).

late as 04:00 LT and estimate that the origin must have been $3000 \mathrm{~km}$ to the west, assuming a drift speed of $100 \mathrm{~m} / \mathrm{s}$. A similar out-of-season EPB was observed at Asmara on 26 December 2001 at 05:00 LT. Its estimated drift speed was $100 \mathrm{~m} / \mathrm{s}$ and, assuming it was seeded at 19:30 LT, it lasted for $9.5 \mathrm{~h}$ and formed $3400 \mathrm{~km}$ to the west of Asmara in West Africa. For this special case the growth rate appeared to be zero.

Makela et al. (2004) do not discuss the decay of EPBs that pass over Hawaii, so we suppose that most formations simply pass their eastern horizon. In Asmara many EPBs do not reach the eastern horizon but instead fade within the field of view. In situ measurements of plasma density from DMSP corroborate the claim that EPBs are uncommon east of Asmara (Huang et al., 2001). At the 1991 peak of the solar activity cycle they reported many EPBs right up to our longitude of $40^{\circ} \mathrm{E}$ and many fewer to the east of the African coastline. This is in good agreement with the longitude studies reported by McClure et al. (1998), where at the longitude of $40^{\circ} \mathrm{E}$, in all cases the occurrence probability drops off rapidly eastward. As reported by Hocke and Tsuda (2001), gravity wave activity is especially frequent at $40^{\circ} \mathrm{E}$ in the tropics. Gravity wave activity is often viewed as a likely candidate for seeding ESF, but Asmara's data suggest that gravity wave activity may be able to induce mixing at ionospheric altitudes, causing the EPBs to fade. The increasing amplitude of gravity waves with altitude leads to wave breaking that results in turbulence and mixing (Andrews et al., 1987). It is not yet possible to trace the waves from the troposphere into the ionosphere, but travelling ionospheric disturbances (TIDs), which are considered to be ionospheric gravity waves, are well known, and it is reasonable to suppose that many originate in the lower atmosphere. 


\subsection{Zonal EPB drift velocities}

Numerous studies of EPB zonal drift velocities have been conducted in recent years. Perhaps the most extensive set of ground-based images to be so analyzed is reported by Terra et al. (2004) from Cachoeira Paulista $\left(22.4^{\circ} \mathrm{S}, 45.0^{\circ} \mathrm{W}\right.$, $17.8^{\circ} \mathrm{S}$ dip) in Brazil. Their data from 109 nights show a decrease of drift speed with increasing local time from a maximum of $136 \mathrm{~m} / \mathrm{s}$ at 21:00 LT to a minimum average of $62 \mathrm{~m} / \mathrm{s}$ at 04:00 LT. Although a detailed comparison is not intended here, similar values are seen in Figs. 7 and 9. Abalde et al. (2004) analyzed OI 630-nm data from 5 nights and found a range of drift speeds between 85 and $185 \mathrm{~m} / \mathrm{s}$ at Sao Jose dos Campos $\left(23.2^{\circ} \mathrm{S}, 45.9^{\circ} \mathrm{W}\right)$, also in Brazil, somewhat faster than ours and faster than those of Terra et al. (2004) in nearby Cachoeira Paulista. Pimenta et al. (2003) analyzed 12 nights of OI 630-nm all-sky EPB images from two imagers to obtain latitude variations in drift speeds. The stations were Cachoeira Paulista and Sao Joao do Cariri $\left(7.4^{\circ} \mathrm{S}\right.$, $\left.36.5^{\circ} \mathrm{W}\right)$, Brazil. They found peak speeds of $160 \mathrm{~m} / \mathrm{s}$ in the 21:00-22:00 LT time period near the magnetic equator with a minimum for the same time period of $120 \mathrm{~m} / \mathrm{s}$. From Hawaii Tinsley et al. (1997) found drift speeds between 40 and $130 \mathrm{~m} / \mathrm{s}$. These various studies serve to show that our determinations are in fair agreement with most all-sky measurements recently reported. Satellite confirmation is given by Immel et al. (2003), who showed that IMAGE FUV images gave speeds between 90 and $200 \mathrm{~m} / \mathrm{s}$ at 21:00 LT and 10 to $120 \mathrm{~m} / \mathrm{s}$ at 03:00 LT, depending upon the F10.7 index.

GPS scintillation measurements of drift speed were reported by Kil et al. (2000) for Cachoeira Paulista, with average speeds over $150 \mathrm{~m} / \mathrm{s}$ at $20: 00 \mathrm{LT}$ and $100 \mathrm{~m} / \mathrm{s}$ at midnight. These speeds were found to increase for stations closer to the magnetic equator by Kil et al. (2002). The Asmara GPS scintillation measurements of drift speed are about the same as those from Cachoeira Paulista and show the same slowing trend through the morning hours. The data used by Kil et al. (2002) at Cachoeira Paulista come from identical Cornell GPS scintillation monitors with the same data processing routines as those used in Asmara, so we suppose this comparison to be especially valuable. The similarity between the Asmara GPS scintillation pattern speeds and the Asmara EPB speeds suggests that the vertical shear of horizontal drift is either absent or small. The altitude assumed for the EPBs was that assumed for the OI 630-nm airglow layer or $280 \mathrm{~km}$. The GPS scintillation altitude has been assumed to be the height of the ionization maximum, assumed here to be $350 \mathrm{~km}$. The gradient implied by Haerendel et al. (1992) on the basis of theory and data lies within the error bars of our two methods.

The winter maximum of drift speeds shown in Fig. 8 has not been reported for EPB or GPS scintillation determinations of zonal ionospheric drift velocity. Nevertheless, it was reported by Pathan and Rao (1996) from 244-MHz FLEETSAT beacon signals at Trivandrum on the magnetic equator in India, though less evident and excluding the early evening hours.

\section{Conclusions}

Observations of EPB and GPS scintillation occurrence and drift speed have been reported for a 9-month period during the recent maximum of solar activity from Asmara in northeast Africa. Our conclusions are enumerated as follows:

1. With its magnetic dip latitude at $7^{\circ} \mathrm{N}$, just south of the EIA crest, there is an abundance of ESF with EPBs appearing on $63 \%$ of nights with possible observing conditions and GPS scintillations of $\mathrm{S}_{4}>0.4$ appearing on $75 \%$ of nights of operation.

2. The occurrence of both EPBs and GPS scintillations is most probable around the equinoxes and least around the winter solstice with a suggestion that the centers of activity are as much as 20 days after the actual equinox dates. The summer solstice behavior is found only from our GPS scintillation data, which indicate that it is at least as deep as the winter solstice minimum. The equinox maxima of occurrence differ from the seasonal dependence at other longitude sectors, confirming the longitude dependence of seasonal EPB occurrence behavior.

3. The earliest sightings of EPBs during equinox periods seem to coincide with the first time that the GPS scintillation $\mathrm{S}_{4}$ index rises to about 0.4 or more. Some EPBs appear to originate at the longitude of Asmara at these times.

4. EPBs generally persist into the morning hours after the GPS scintillation index has reduced to below 0.4, indicating that the small scale structure dissipates more quickly than the large scale structure.

5. EPBs during the morning hours always come from the western horizon. They expand as they drift and are often seen to fade before they reach the eastern horizon. We suggest that gravity wave activity, known to be abundant at Asmara's longitude, may induce mixing at ionospheric altitudes, which could possibly explain why Asmara might be the terminus for many EPBs.

6. Zonal ionospheric drift speeds were deduced from both EPB drift speeds and GPS scintillation speeds. Both methods showed the decrease with local time reported by others, and both showed a winter maximum in drift speeds.

7. Average drift speeds measured for EPBs and for GPS scintillations were between 100 and $120 \mathrm{~m} / \mathrm{s}$ for much of the year, but late morning speeds in May were about $40 \mathrm{~m} / \mathrm{s}$, our minimum. 
Acknowledgements. The authors are grateful to G. G. Shepherd for the loan and support of the York University all-sky imager, and to G. Ogubazghi for encouragement and the use of University of Asmara facilities. GPS scintillation monitor development at Cornell is funded by the U.S. Office of Naval Research under grant N0001404-1-0105. RHW's work at Cornell is supported by the U.S. National Science Foundation under grant ATM-0404706.

Topical Editor M. Pinnock thanks two referees for their help in evaluating this paper.

\section{References}

Abalde, J. R., Fagundes, P. R., Sahai, Y., Pillat, V. G., Pimenta, A. A., and Bittencourt, J. A.: Height-resolved ionospheric drifts at low latitudes from simultaneous OI $777.4 \mathrm{~nm}$ and OI $630.0 \mathrm{~nm}$ imaging observations, J. Geophys. Res., 109, A11308, doi:10.1029/2004JA010560, 2004.

Andrews, D. G., Holton, J. R., and Leovy, C. B.: Middle Atmosphere Dynamics, Academic Press, Orlando, p. 193, 1987.

Beach, T. L. and Kintner, P. M.: Development and use of a GPS ionospheric scintillation monitor, IEEE Trans. Geosci. Remote Sens., 39(5), 918-928, 2001.

Burke, W. J., Huang, C. Y., Gentile, L. C., and Bauer, L.: Seasonallongitudinal variability of equatorial plasma bubbles, Ann. Geophys., 22, 3089-3098, 2004.

Criswick, J. R.: An all-sky imager for observing airglow activity in the upper atmosphere, M.Sc. Thesis, York University, Toronto, Canada, 1994.

Fejer, B. G., Farley, D. T., Gonzalez, C. A., Woodman, R. F., and Calderon, C.: F region east-west drifts at Jicamarca, J. Geophys. Res., 86, 215-218, 1981.

Gentile, L. C., Burke, W. J., and Rich, F. J.: A global climatology for equatorial plasma bubbles in the topside ionosphere, Ann. Geophys., 24, 163-172, 2006.

Haerendel, G., Eccles, J. V., and Cakir, S.: Theory for modeling the equatorial evening ionosphere and the origin of the shear in the horizontal plasma flow, J. Geophys. Res., 97(A2) 1209-1223, 1992.

Hocke, K. and Tsuda, T.: Gravity waves and ionospheric irregularities over tropical convection zones observed by GPS/MET radio occultation, Geophys. Res. Lett., 28(14), 2815-2818, 2001.

Huang, C. Y., Burke, W. J., Machuzak, J. S., Gentile, L. C., and Sultan, P. J.: DMSP observations of equatorial plasma bubbles in the topside ionosphere near solar maximum, J. Geophys. Res., 106(A5), 8131-8142, 2001.

Immel, T. J., Mende, S. B., Frey, H. U., and Peticolas, L. M.: Determination of low latitude plasma drift speeds from FUV images, Geophys. Res. Lett., 30(18), 1945, doi:10.1029/2003GL017573, 2003.

Kil, H., Kintner, P. M., de Paula, E. R., and Kantor, I. J.: Global Positioning System measurements of the ionospheric zonal apparent velocity at Cachoeira Paulista in Brazil, J. Geophys. Res., 105(A3), 5317-5327, 2000.

Kil, H., Kintner, P. M., de Paula, E. R., and Kantor, I. J.: Latitudinal variation of scintillation activity and zonal plasma drifts in South America, Radio Sci., 37(1), doi:10.1029/2001RS002468, 2002.

Kil, H., DeMajistre, R., and Paxton, L. J.: F-region plasma distribution seen from TIMED/GUVI and its relation to the equatorial spread F activity, Geophys. Res. Lett., 31, L05810, doi:10.1029/2003GL018703, 2004.

Ledvina, B. M., Kintner, P. M., and de Paula, E. R.: Understanding spaced-receiver zonal velocity estimation, J. Geophys. Res., 109(A10), doi:10.1029/2004JA010489, 2004.

Makela, J. J., Ledvina, B. M., Kelley, M. C., and Kintner, P. M.: Analysis of the seasonal variations of equatorial plasma bubble occurrence observed from Haleakala, Hawaii, Ann. Geophys., 22, 3109-3121, 2004.

Maruyama, T. and Matuura, N.: Global occurrence probability of spread echoes based on ISS-b observation, J. Radio Res. Lab. Jpn., 27, 201-216, 1980.

Maruyama, T. and Matuura, N.: Longitudinal variability of annual changes in activity of equatorial spread $\mathrm{F}$ and plasma bubbles, J. Geophys. Res., 89, 10 903-10 912, 1984.

McClure, J. P., Singh, S., Bamgboye, B. K., Johnson, F. S., and Kil, H.: Occurrence of equatorial $F$ region irregularities: Evidence for tropospheric seeding, J. Geophys. Res., 103(A12), 29119 $29135,1998$.

Mukherjee, G. K.: Studies of equatorial F-region depletions and dynamics using multiple-wavelength nightglow imaging, J. Atmos. Solar-Terr. Phys., 65, 379-390, 2003.

Pathan, B. M., and Rao, D. R. K.: Seasonal and solar cycle association of zonal drifts of ionospheric plasma irregularities in the Indian equatorial region, Ann. Geophys., 14, 297-303, 1996.

Pimenta, A. A., Bittencourt, J. A., Fagundes, P. R., Sahai, Y., Burit, R. A., Takahashi, H., and Taylor, M. J.: Ionospheric plasma bubble zonal drifts over the tropical region: A study using OI $630 \mathrm{~nm}$ emission all-sky images, J. Atmos. Solar-Terr. Phys., 65, 1117-1126, 2003.

Terra, P. M., Sobral, J. H. A., Abdu, M. A., Souza, J. R., and Takahashi, H.: Plasma bubble zonal velocity variations with solar activity in the Brazilian region, Ann. Geophys., 22, 3123-3128, 2004.

Tinsley, B. A., Rohrbaugh, R. P., Hanson, W. B., and Broadfoot, A. L.: Images of transequatorial $F$ region bubbles in 630- and 777nm emissions compared with satellite measurements, J. Geophys. Res., 102, 2057-2078, 1997.

Tsunoda, R. T.: Control of the seasonal and longitudinal occurrence of equatorial scintillations by the longitudinal gradient in integrated E region Pedersen conductivity, J. Geophys. Res., 90, 447-456, 1985.

Valladares, C. E., Sheehan, R., Basu, S., Kuenzler, H., and Espinoza, J.: The multi-instrumented studies of equatorial thermosphere aeronomy scintillation system: Climatology of zonal drifts, J. Geophys. Res., 101(A12), 26 839-26 850, 1996.

Watanabe, S. and Oya, H.: Occurrence characteristics of lowlatitude ionospheric irregularities observed by impedance probe onboard the Hinotori satellite, J. Geomagn. Geoelectr., 38, 125 $149,1986$.

Wiens, R. H., Habtemichael, S., Andemariam, F., Welday, K., Criswick, J., Brown, S., and Sargoytchev, S.: Brightness variations of the northern $630 \mathrm{~nm}$ intertropical arc and the midnight pressure bulge over Eritrea, Ann. Geophys., 22, 3251-3259, 2004.

Yeh, K. C. and Liu, C. H.: Radio wave scintillations in the ionosphere, Proc. IEEE, 70(4), 324-360, 1982. 